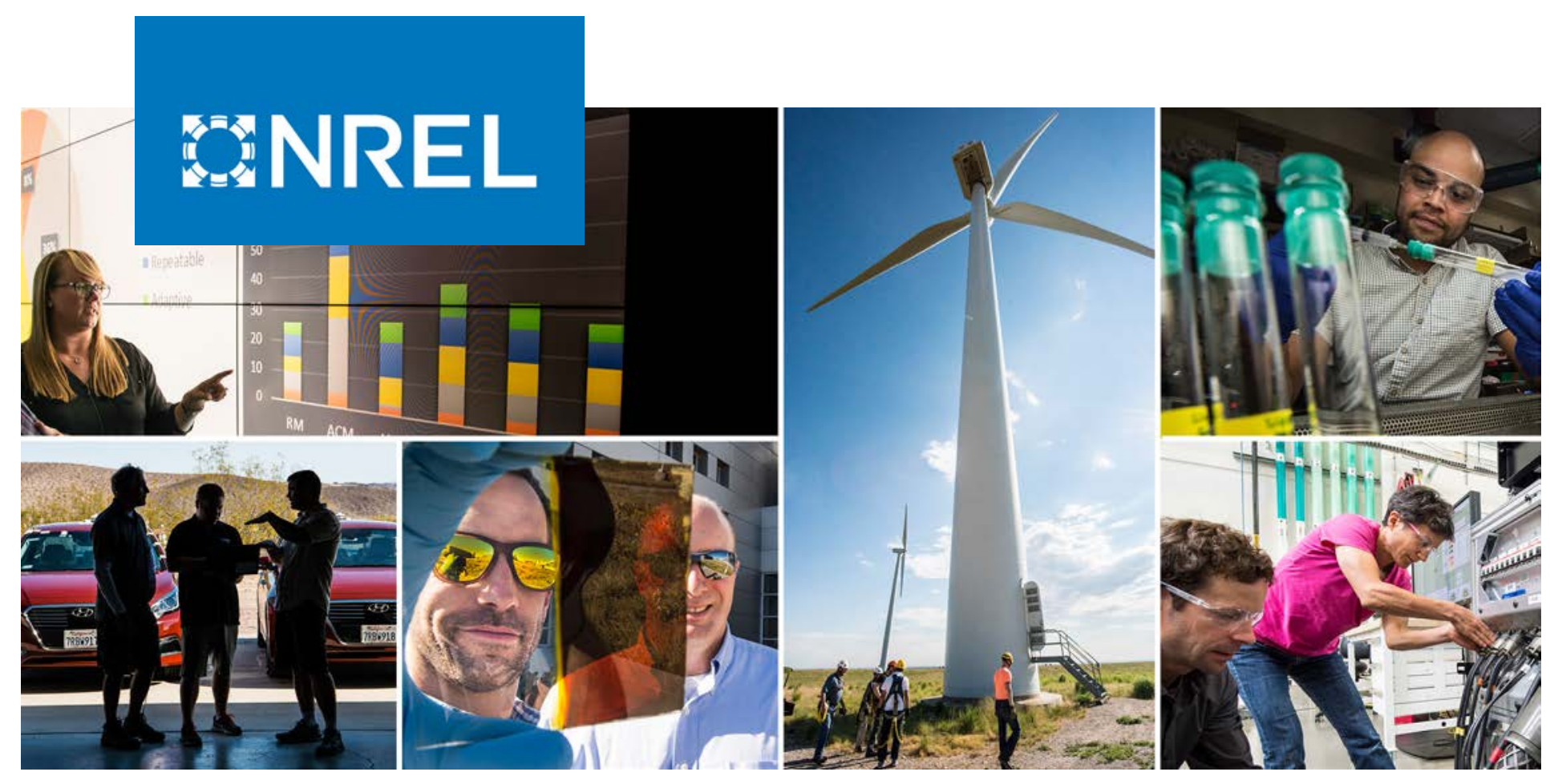

\title{
Online Static Load Model Estimation in Distribution Systems
}

\section{Preprint}

Hongda Ren, ${ }^{1}$ Venkat Krishnan, ${ }^{2}$ Yingchen Zhang, ${ }^{2}$ and Noel N. Schulz ${ }^{1}$

${ }^{1}$ Washington State University

${ }^{2}$ National Renewable Energy Laboratory

Presented at the 2019 IEEE 28th International Symposium on Industrial Electronics (IEEE ISIE)

Vancouver, Canada

June 12-14, 2019

NREL is a national laboratory of the U.S. Department of Energy

Office of Energy Efficiency \& Renewable Energy

Operated by the Alliance for Sustainable Energy, LLC

This report is available at no cost from the National Renewable Energy Laboratory (NREL) at www.nrel.gov/publications.

\section{Conference Paper}

NREL/CP-5D00-73228

August 2019 


\title{
GNREL
}

\section{Online Static Load Model Estimation in Distribution Systems}

\section{Preprint}

\author{
Hongda Ren, ${ }^{1}$ Venkat Krishnan, ${ }^{2}$ Yingchen Zhang, ${ }^{2}$ \\ and Noel N. Schulz ${ }^{1}$ \\ ${ }^{1}$ Washington State University \\ ${ }^{2}$ National Renewable Energy Laboratory
}

\section{Suggested Citation}

Ren, Hongda, Venkat Krishnan, Yingchen Zhang, and Noel N. Schulz. 2019. Online Static

Load Model Estimation in Distribution Systems: Preprint. Golden, CO: National

Renewable Energy Laboratory. NREL/CP-5D00-73228.

https://www.nrel.gov/docs/fy19osti/73228.pdf.

(C) 2019 IEEE. Personal use of this material is permitted. Permission from IEEE must be obtained for all other uses, in any current or future media, including reprinting/republishing this material for advertising or promotional purposes, creating new collective works, for resale or redistribution to servers or lists, or reuse of any copyrighted component of this work in other works.

NREL is a national laboratory of the U.S. Department of Energy Office of Energy Efficiency \& Renewable Energy Operated by the Alliance for Sustainable Energy, LLC

This report is available at no cost from the National Renewable Energy Laboratory (NREL) at www.nrel.gov/publications.

Contract No. DE-AC36-08GO28308
Conference Paper

NREL/CP-5D00-73228

August 2019

National Renewable Energy Laboratory 15013 Denver West Parkway Golden, CO 80401

303-275-3000 • www.nrel.gov 


\section{NOTICE}

This work was authored in part by the National Renewable Energy Laboratory, operated by Alliance for Sustainable Energy, LLC, for the U.S. Department of Energy (DOE) under Contract No. DE-AC36-08GO28308. Funding provided by U.S. Department of Energy Office of Energy National Renewable Energy Laboratory's Laboratory Directed Research and Development Program (LDRD) and the U.S. Department of Energy's Office of Electricity Delivery and Energy Reliability (OE). The views expressed herein do not necessarily represent the views of the DOE or the U.S. Government. The U.S. Government retains and the publisher, by accepting the article for publication, acknowledges that the U.S. Government retains a nonexclusive, paid-up, irrevocable, worldwide license to publish or reproduce the published form of this work, or allow others to do so, for U.S. Government purposes.

This report is available at no cost from the National Renewable Energy Laboratory (NREL) at www.nrel.gov/publications.

U.S. Department of Energy (DOE) reports produced after 1991 and a growing number of pre-1991 documents are available free via www.OSTI.gov.

Cover Photos by Dennis Schroeder: (clockwise, left to right) NREL 51934, NREL 45897, NREL 42160, NREL 45891, NREL 48097, NREL 46526.

NREL prints on paper that contains recycled content. 


\section{Online Static Load Model Estimation in Distribution Systems}

\author{
Hongda Ren, Student Member, IEEE \\ Noel N. Schulz, Fellow, IEEE \\ School of Electrical Engineering and Computer Science \\ Washington State University, Washington, USA \\ hongda.ren@wsu.edu
}

\author{
Venkat Krishnan, Senior Member, IEEE \\ Yingchen Zhang, Senior Member, IEEE \\ Power System Engineering Center (PSEC) \\ National Renewable Energy Laboratory, CO, USA \\ Venkat.Krishnan@nrel.gov
}

\begin{abstract}
This paper focuses on parameter identification for time-varying voltage-dependent polynomial load models in distribution systems-namely, the constant impedance $(Z)$, constant current (I), and constant power (P). The paper assumes the presence of measurements at the distribution nodes from various sources, each at varying temporal resolutions. The leastsquares estimation method with bounded variables is used to convert the measurements into ZIP parameter estimations for loads at various locations on the feeder. The results and discussions focus on some of the estimation issues faced in a distribution system caused by varying voltage sensitivities of loads along a feeder, measurement resolution, impact of window size and sampling rate of measurement data. Finally, the paper uses dynamic simulations data (synthetic high-resolution measurements) to further improve the estimation results.
\end{abstract}

Index Terms-- Least-squares estimation, load parameters, voltage measurements, dynamics, distribution systems.

\section{INTRODUCTION}

Static load models are typically represented by polynomial functions of node voltages and, at times, with additional terms capturing frequency dependence [1]. Typically, the most common polynomial relationships fall under three categories: constant impedance $(Z)$, for which the power varies proportional to the square of voltage; constant current (I), for which power variations are directly proportional to the voltage; and constant power load (invariant to voltage changes). Every load component exhibits a mixture of such voltage-dependent behavior. Bokhari et. al. experimentally verified the coefficients of most commonly found residential, commercial, and industrial load types in [2]. Identifying the right load composition has a direct impact on system stability assessments and planning, and therefore a number of transmission-level planning studies have delved deeper into validating load models using utility surveys and phasor measurement unit (PMU) measurements at substations [3]. For transient stability assessment [4], it becomes essential to also capture the secondary effects of load types on voltages, and therefore the Western Electricity Coordinating Council developed a composite load model that captures the dynamic behavior of induction motors and electronic loads in addition to the static ZIP models [5]. Chassin et. al. developed a load modeling tool that can estimate the transmission-level aggregated load composition for various regions of the country subject to seasonal and time of the day variations [6].
With the rich background of work at the aggregated transmission level and studies needing higher fidelity models for distribution networks (either for coupled transmission-anddistribution or distribution-only analysis), there is a challenge to develop accurate models for distribution networks. The increased customer activity and the ever-changing mixture of load compositions need to be captured. Moreover, given the need to control voltages within the ANSI limits, there is a high value for estimating the changing load composition and forecasting its impact on voltage in real time. Such a paradigm will include online load model estimation using real-time measurements and state estimation data. A sliding window of data (Fig. 2b) will be used for continuous estimation and update of load models [7]. To move toward an online paradigm, this paper focusses on the load model estimation methods for distribution systems considering different types of measurement data.

In the open literature, genetic algorithm and nonlinear Levenberg-Marquardt methods have been combined to identify parameters for aggregated ZIP and induction motor loads [8]. For online applications, an event-driven estimation is applied, in which voltage excursion events are detected to estimate the composite load models with constant ZIP and dynamic loads; whereas without events, only ZIP static models are estimated. The least-squares estimation (LSE) method with adjustable sliding window is used for parameter identification in real time [1]. Banerjee et. al. [9] proposed estimating the confidence factor to quantify the uncertainties in the load model estimation. In all of these works, the estimation methods had performance issues under steady-state voltage measurements and required lower voltage phenomena to differentiate between load types. A relevant question for distribution systems is: Can such voltage dynamics come from phenomena such as sudden load changes, switching, and tap changing, in addition to fault scenarios? In the absence of sensor data from real distribution networks, can dynamic simulations [10] help synthesize high-resolution measurement data needed for online load model estimation?

This paper looks into some of these challenges in load model estimation for distribution networks. The paper initially uses synthetic measurement data from quasi-static time-series (QSTS) simulations on an IEEE 13-node feeder model, where the voltages are controlled within the ANSI limits. The LSE methods are applied for all time-varying loads in a distribution 
feeder, thereby also capturing the mutual impacts between load variations. For online applications, the paper also investigates time-varying loads in a feeder and studies the effects of locations, window size, sampling rate, and timevarying load profile on estimation results. Finally, the paper uses dynamic simulations of distribution networks to capture the lower voltage transients, thereby demonstrating the use of very high-resolution synthetic measurement data that can improve the accuracy of the load model estimation method.

\section{ZIP LOAD MOdEL Estimation: STEADY-STATE DATA}

The IEEE 13-node system (with phase imbalances), Fig. 1, was used, and 24-hour QSTS simulation was performed to create synthetic measurement data. Measurement errors were not considered. The loads (circled in Fig. 1) located in the middle (load 671 (Delta-ABC)) and edges of the feeder (loads 611 (Wye-C), 652 (Wye-A), 675 (Wye-ABC), 634 (Wye$\mathrm{ABC}$ ), and 645 (Wye-B)) were selected to illustrate the location effects on estimation results. A time-varying load profile, as shown in Fig. 2a, was considered. Simulation results (a proxy for sensor data) were used in a sliding window manner (i.e., a constant "window size" of sensor data moved by "move step," as shown in Fig. 2b) for the online estimation (with "window size-move step" as data overlap between successive estimation windows).

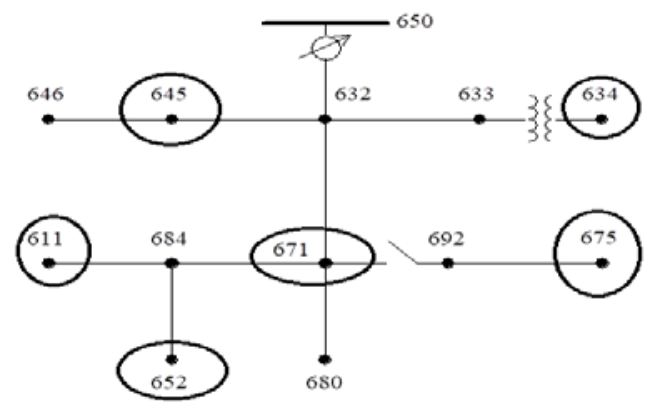

Fig. 1 Multiple time-varying loads in IEEE 13-bus system

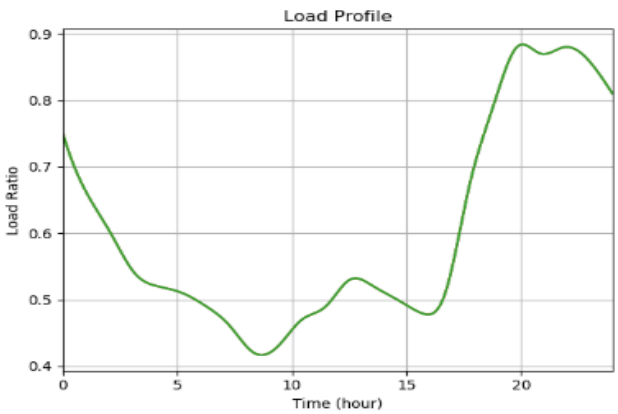

Fig. 2a

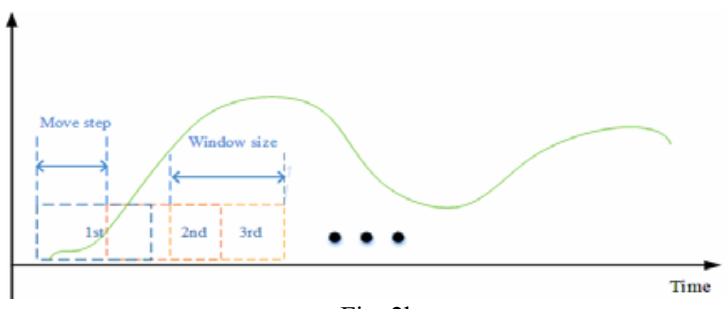

Fig. 2b

Fig. 2 (a) Load profile, (b) sliding windows for online estimation

\section{A. Static Load Models}

Eq. (1) and (2) indicate the modeling of constant impedance $(\mathrm{Z})$, constant current $(\mathrm{I})$, and constant power $(\mathrm{P})$ loads, making up a polynomial function of power versus voltage. $\mathrm{P}_{0}, \mathrm{Q}_{0}$ are the base active and reactive power of the load at rated voltage $\left(\mathrm{V}_{0}\right) . \mathrm{Z}_{\mathrm{P}}, \mathrm{I}_{\mathrm{P}}, \mathrm{P}_{\mathrm{P}}, \mathrm{Z}_{\mathrm{Q}}, \mathrm{I}_{\mathrm{Q}}$, and $\mathrm{P}_{\mathrm{Q}}$ are the $\mathrm{ZIP}$ coefficients for the active and reactive power, respectively. The ZIP coefficients have two constraints: 1) $\mathrm{Z}+\mathrm{I}+\mathrm{P}=1$, and 2) $\mathrm{Z}, \mathrm{I}, \mathrm{P} \geq 0$.

$$
\begin{aligned}
& P=P_{0}\left[Z_{P}\left(\frac{V}{V_{0}}\right)^{2}+I_{P}\left(\frac{V}{V_{0}}\right)+P_{P}\right] \\
& Q=Q_{0}\left[Z_{Q}\left(\frac{V}{V_{0}}\right)^{2}+I_{Q}\left(\frac{V}{V_{0}}\right)+P_{Q}\right]
\end{aligned}
$$

\section{B. Least-Squares Estimation}

The LSE method is a linear parameter estimation method to minimize errors between outputs of model estimation with measurements [11]. Eq. (3) and (4) illustrate the parameter estimation by using LSE. The parameter vectors $[\mathrm{Z}, \mathrm{I}, \mathrm{P}]$ contain ZIP coefficients. The A matrix is built with voltage measurements V1, V2, and V3 and the power measurements $\mathrm{P} 1, \mathrm{P} 2$, and $\mathrm{P} 3$, by phase. $\mathrm{V} 0$ and $\mathrm{P} 0$ are the rated voltage and power of a load. LSE with bounds on the variables are applied to meet the ZIP parameter constraints discussed here.

$$
\begin{gathered}
A * Z I P=\left[\begin{array}{lll}
\left(\frac{V_{1}}{V_{0}}\right)^{2} & \left(\frac{V_{1}}{V_{0}}\right) & 1 \\
\left(\frac{V_{2}}{V_{0}}\right)^{2} & \left(\frac{V_{2}}{V_{0}}\right) & 1 \\
\left(\frac{V_{3}}{V_{0}}\right)^{2} & \left(\frac{V_{3}}{V_{0}}\right) & 1
\end{array}\right]\left[\begin{array}{l}
Z \\
I \\
P
\end{array}\right]=\left[\begin{array}{l}
\frac{P_{1}}{P_{0}} \\
\frac{P_{2}}{P_{0}} \\
\frac{P_{3}}{P_{0}}
\end{array}\right] \\
\left.\mid \begin{array}{l}
Z \\
I \\
P
\end{array}\right]=\left(A^{T} A\right)^{-1} A^{T} \cdot P_{\text {measpu }}
\end{gathered}
$$

The mean absolute percentage error (MAPE), Eq. (5), evaluates difference between estimation parameters and real values [12]. $\mathrm{A}_{\mathrm{i}}$ is the actual value, $\mathrm{F}_{\mathrm{i}}$ is the estimation, and $\mathrm{N}$ is the total number of estimation points in the process.

$$
\text { MAPE }=\frac{1}{N} \sum_{i=1}^{N} \mid \frac{A_{i}-F_{i}}{A_{i}}
$$

\section{Preliminary Online LSE Results and Sampling Rates}

The function 'scipy.optimize.lsq_linear' with optimization method setting as Trust Region Reflective algorithm is used in the parameter estimation in Python [13]. A sliding window of $1.5 \mathrm{~h}$ (6 samples) was used, with data sampled at 15-min intervals. Between two sliding windows, a 15-min moving step was used. The 15-min interval depicts the current state-of the-art measurements from advanced metering infrastructure (AMI) that is deployed in the distribution systems. For each sampling window, the rated power (P0*LoadMult) and voltage (V0) are used as the base. Then, based on the sampled data in that window, the ZIP estimates are updated.

Six different combinations of ZIP were simulated, and LSE was used to estimate the loads. As shown in Table I, the LSE method was able to estimate the constant $\mathrm{Z}$ and constant 
P loads fairly accurately with active power and reactive power measurements; however, inaccuracy showed in estimating the constant I load model for the active or reactive portion of load. Figs. 3 and 4 show the estimation results for selected cases at load node 671 (hub node). The estimation results in the figures follow this convention: red lines are real ZIP parameters, and black lines are LSE estimation results (y-axis) updated at regular intervals. All x-axes show sliding windows.

TABLE I PRELIMINARY CONSTANT ZIP LOAD ESTIMATION RESUltS

\begin{tabular}{|c|c|c|c|c|}
\hline Case No & P & Q & Result P & Result Q \\
\hline $\mathbf{1}$ & Z & Z & Good & Good \\
\hline $\mathbf{2}$ & Z & I & Good & Inaccurate \\
\hline $\mathbf{3}$ & Z & P & Good & Good \\
\hline $\mathbf{4}$ & I & Z & Inaccurate & Good \\
\hline $\mathbf{5}$ & P & Z & Good & Good \\
\hline $\mathbf{6}$ & I & I & Inaccurate & Inaccurate \\
\hline
\end{tabular}
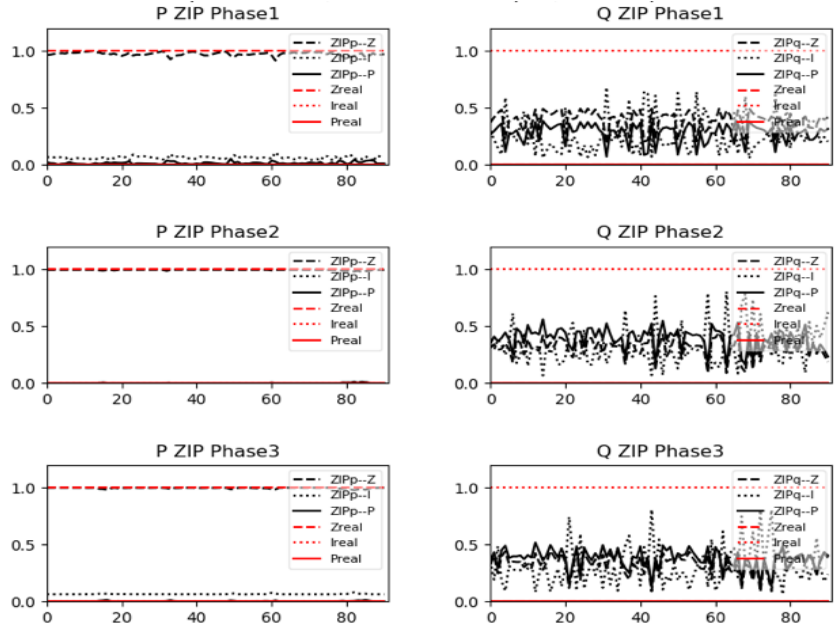

Fig. 3 Case 2 results: constant $\mathrm{Z}$ for $\mathrm{P}$ and constant I for Q load ( $y$-axis: ZIP proportion; $x$-axis: estimation windows)
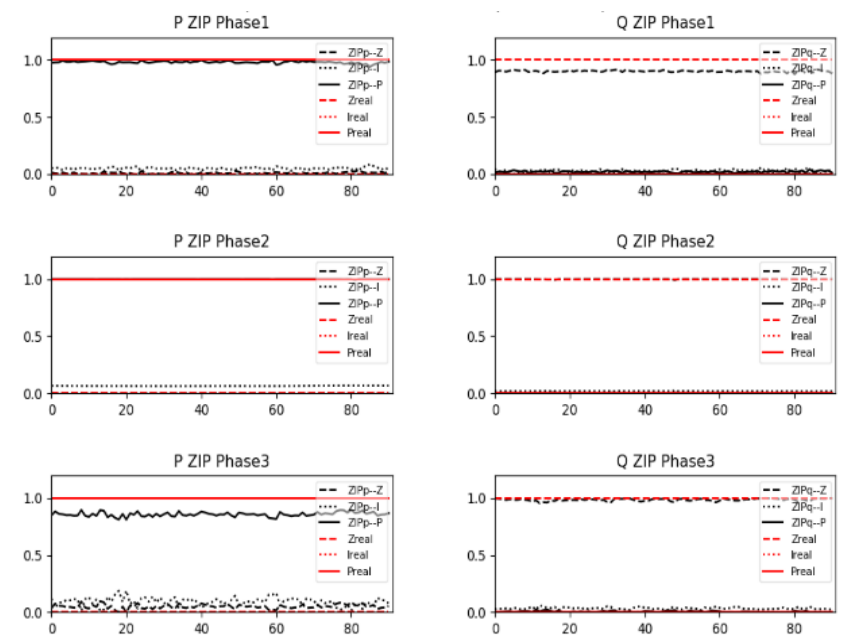

Fig. 4 Case 5 results: constant $\mathrm{P}$ for real power $(\mathrm{P})$, constant $\mathrm{Z}$ for reactive power (Q) load (y-axis: ZIP proportion, $x$-axis: estimation windows)

To confirm the performance in Table I (experimented for one hub location, 671), the loads at multiple feeder end locations (circled in Fig. 1) were modified to constant P/Power and $\mathrm{Z} /$ Impedance models for active $(\mathrm{P})$ and reactive power (Q), respectively. Further, in the 24-hour simulation, an abrupt load model change was introduced in hour 6 to see the impact of an event on the estimation. The load model for the active power was changed from $100 \%$ constant $P$ to $80 \%$ constant $P$ and $20 \%$ constant $Z$, and for the reactive power changed from $100 \%$ constant $Z$ to $20 \%$ constant $Z$ and $80 \%$ constant $P$.

Fig. 5 shows the estimation results for all the selected feeder end loads (three phases, where applicable) for the ZP load combination. Despite the abrupt load model change, the online estimation settles very close to the correct values for all the loads. Further, note that the sampling rate in Fig. 5 is at 30 $\mathrm{s}$ with a 75-min sample window (compared to the earlier 15min window), which enabled estimating the ZP proportions with better accuracy than lower sampling rates.
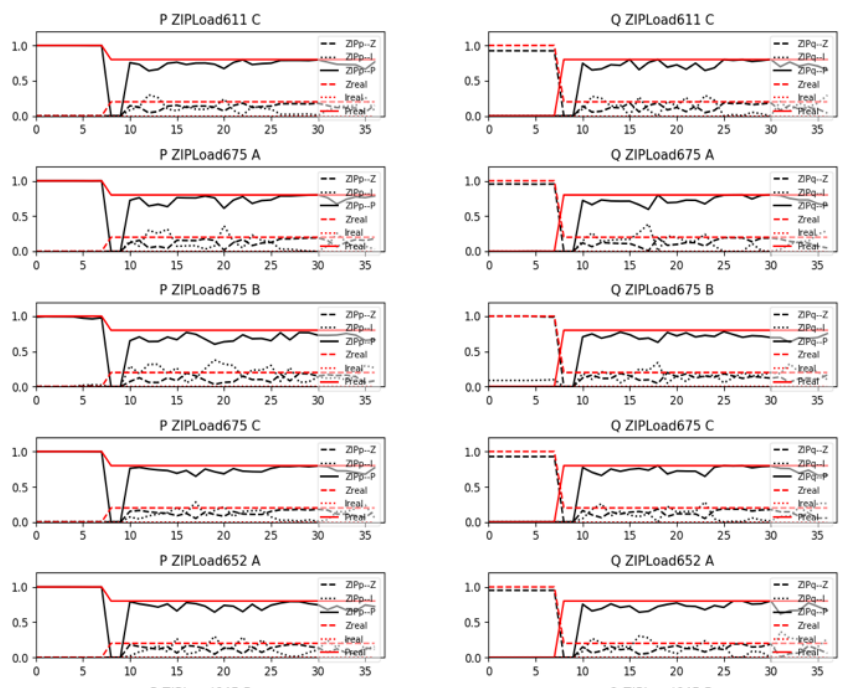
Q ZIPLoad645 B
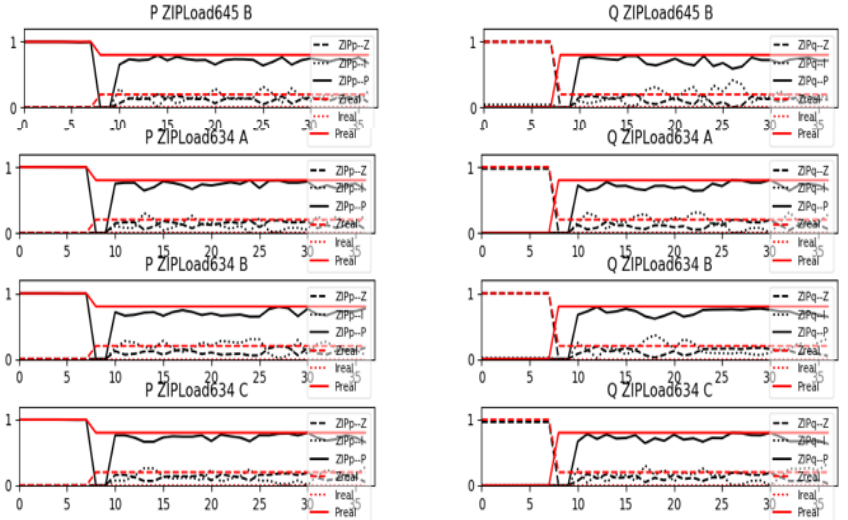

Fig. 5 Time-varying online ZP model estimation: feeder end loads (y-axis: ZIP proportion, $x$-axis: estimation windows)

The increased sampling rate signifies the importance of deploying high-resolution sensors in distribution systems, which can help improve the load model estimation accuracy. Through sensitivity studies on sampling rates (15 min, $60 \mathrm{~s}, 30$ $\mathrm{s}, 5 \mathrm{~s}$ ), it was found that for the same sliding window size, the higher the sampling rate of measurements, the lower the MAPE (i.e., a higher estimation accuracy). This is because a higher sampling rate is able to capture higher time-varying information, especially when there is an event (such as the 
load model change simulated at hour 6). For online applications, the sampling rates could be adjusted dynamically based on the voltage events detected.

\section{Constant I Load: Steady-State-Based LSE Improvement}

Table I shows that whenever the constant I is included in the ZIP combination, the estimation accuracy suffers. This section further investigates the constant I load.

\section{1) Higher Samping Rate}

Fig. 6 shows that with higher sampling rates of measurements (60 s vs. $5 \mathrm{~s}$, during 240-min window), the LSE is able to better estimate the constant I load portion; however, much improvement is still desired (see phase $2 / \mathrm{B}$ for 5 -s estimation).
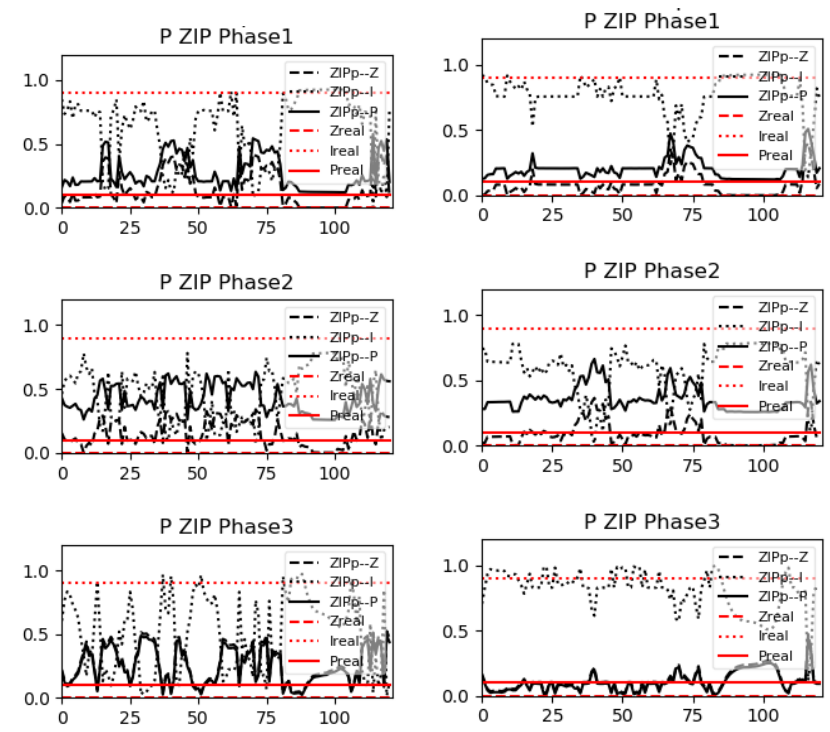

Fig. 6 LSE results for constant I: (left) 60-s and (right) 5-s sample rate (load 671 with $90 \%$ I and $10 \%$ constant $\mathrm{P}$ for active power) (y-axis: ZIP proportion; $x$-axis: estimation windows)

\section{2) Individual Component LSE Error Estimation}

In this subsection, we consider only the $100 \%$ constant I scenario (the last case in Table I) and perform two types of estimation: Type 1: conventional LSE, where all components are considered during estimation ( $a L S E)$, as shown in Eq. (3); and Type 2: LSE for each individual component (iLSE). As shown in (6), each $i L S E$ equation will provide the respective component error with respect to actual measured values. The reciprocals of errors (7) will be used to estimate the ZIP proportions (subject to (8)).

$$
\begin{aligned}
& \text { Constant } Z \text { error }=A[:, 1]-P_{\text {meas_pu }} \\
& \text { Constant } I \text { error }=A[:, 2]-P_{\text {meas_pu }} \\
& \text { Constant } P \text { error }=A[:, 3]-P_{\text {meas } p u} \\
& \frac{1}{\text { Constant } Z \text { error }}: \frac{1}{\text { Constant } I \text { error }}: \frac{1}{\text { Constant } P \text { error }} \approx Z: I: P \\
& Z_{P}+I_{P}+P_{P}=1
\end{aligned}
$$

Fig. 7 shows the comparison between the proposed $i L S E$ and $a L S E$ methods for real power load (with 15-min window data at 5-s intervals). Although $a L S E$ (left-side figures) has trouble differentiating the I load (MAPE $>70)$, the proposed $i L S E$ method (right-side figures) is able to estimate the I load more accurately (MAPE $<10)$.
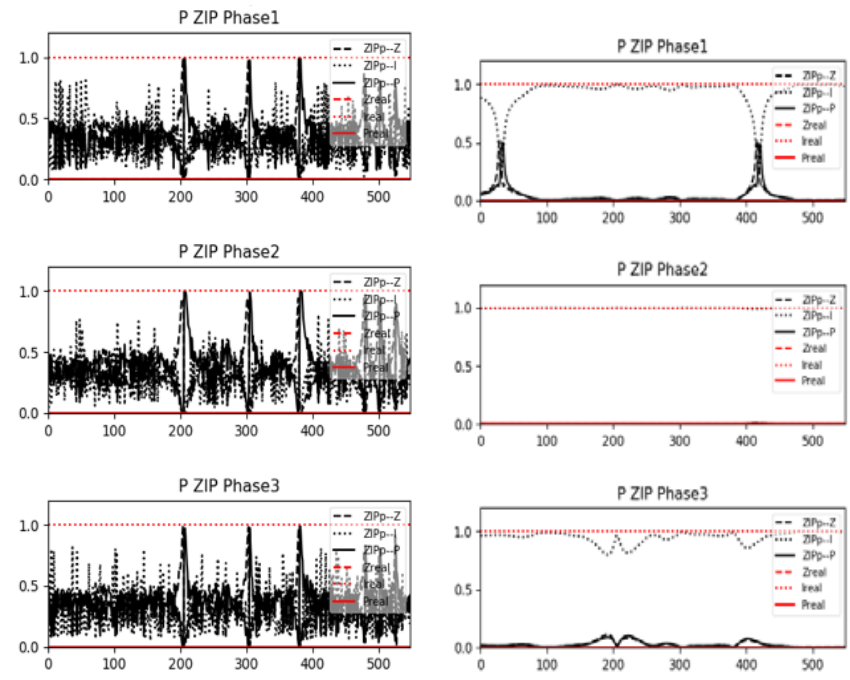

Fig. 7 Load 671, constant I: (left) conventional LSE ( $a L S E)$ and (right) individual component LSE ( $i L S E)$, with 5-s, 15-min window data ( $y$-axis: ZIP proportion; $x$-axis: estimation windows)

The method discussed will work specifically for situations when a load has $100 \% \mathrm{Z}$ or I or P load, thereby aiding the use of $i L S E$ for each component and using their error reciprocals (a distance measure to actual data). A hybrid estimation model (with $S L S E$ and $i L S E$ ) can be conceived, where $i L S E$ might be used when $a L S E$ results are highly fluctuating. Nevertheless, this hybrid model does not solve the estimation issue when a combination of ZIP (or ZI or PI) load model is available.

\section{ZIP LOAD MODEL ESTIMATION: DYNAMIC DATA}

Section II used steady-state data (i.e., simulated 15-min AMI or 5-s SCADA data) for estimations and discussed the extent to which the LSE-based estimations could be improved. This section investigates the use of dynamic simulation data $(<1 \mathrm{~s})$ to mimic the data availability from the proliferation of microPMUs and digital disturbance recorders under grid modernization futures. Fig. 8, which shows the power-voltage relationship as portrayed by the ZIP load model ((1) and (2)), illustrates why capturing voltage dynamics will be important to improve the estimation, even for static load models, specifically for the constant I load. Under normal steady-state conditions, the voltages of the loads vary between ANSI limits of 0.95 p.u. and 1.05 p.u., which are tightly controlled by regulators and capacitors in distribution feeders. In that range, the constant impedance load shows nearly linear relation to voltage, which is similar to the constant current load. Given that LSE minimizes the estimation error, the constant I load could be used to substitute for the combination of constant $\mathrm{P}$ and $Z$ (i.e., average of $P$ and $Z$, because all three loads seem to behave linearly in the band of $0.95-1.05$ p.u.), and vice versa. For instance, in the scenario with $100 \%$ constant I load, the MAPE from LSE is $10 \mathrm{e}^{-7}$ for a combination of ZIP parameters, whereas the actual constant I estimate is $10 \mathrm{e}^{-4}$ MAPE. In other words, even though the actual load is $100 \%$ constant I, the LSE tends to use a combination of ZIP to attain 
a lower MAPE. As discussed, increasing the sampling resolution and using $i L S E$ improved the estimates to a certain extent, but to further improve them, it will be necessary to capture lower voltage phenomena where the ZIP components might be well differentiated, as seen in Fig. 8 (e.g., 0.4-0.9 V).

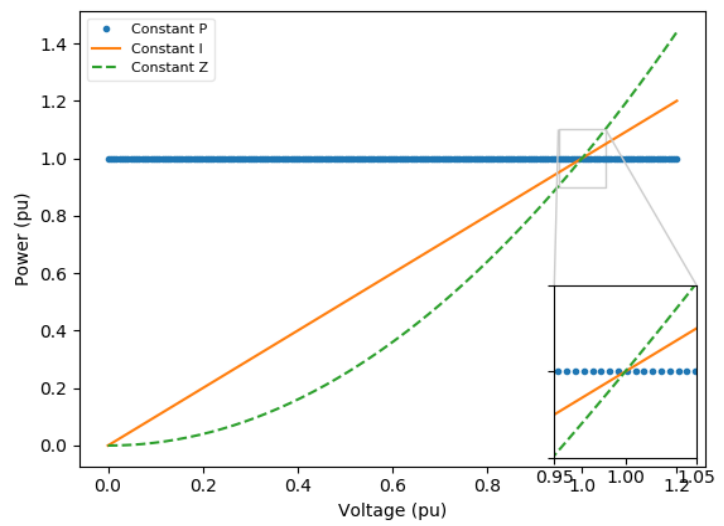

Fig. 8 Voltage and power relation for ideal static load model

\section{A. Dynamic simulation for high-resolution measurements}

The delta mode capability in the GridLAB-D tool is used to simulate distribution system models with time steps less than $1 \mathrm{~s}$, thereby capturing the impact of machine dynamics and generator controls [14]. GridLAB-D is capable of making the transition from quasi-steady-state simulation (QSTS) to delta mode for dynamic simulations. To use voltage dynamics for better estimations in this paper, a Woodward diesel governor (DEGOV1) with a simplified exciter system has been added to a 1.67-MW diesel generator at node 692 [10], [15]. The simulation time step is $10 \mathrm{~ms}$, and a step load change at node 671 is the event that will initiate a voltage transient (a typical event in distribution networks). Fig. 9 shows the post-event simulation response $(\mathrm{P}, \mathrm{Q}$, and $\mathrm{V})$, which captures lower voltage oscillations at the load node.

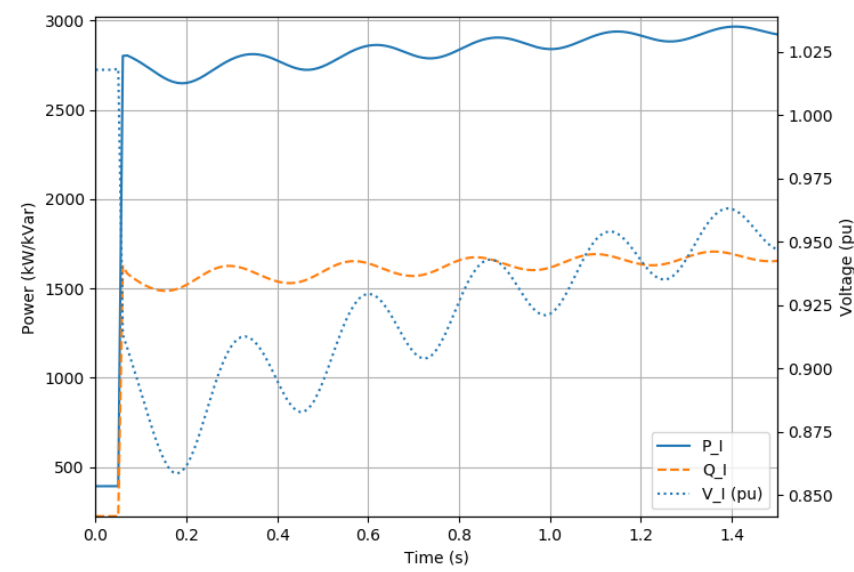

Fig. 9 Dynamic simulation response: $100 \%$ constant I load

Note the use of a step load change event instead of the typical load model estimation practices (at the transmission level) of using post-fault dynamics. Use of the load changerelated transient data in distribution systems will become more relevant under grid modernization futures with higher penetrations of behind-the-meter distributed energy resources, which will cause more frequent random net load changes.

\section{B. LSE-Based ZIP Estimation Using Dynamic Data}

To perform load model estimation using dynamic data, the data shown in Fig 9 was sampled with the time interval of 10 ms during a window of 0.3 s (i.e., 30 samples), with an overlap of $50 \mathrm{~ms}$ (5 samples) across successive sliding windows. Fig. 10 shows the estimation results for the $100 \%$ constant I scenario during a period of $1.2 \mathrm{~s}$. The estimation accuracies are higher periodically when $100 \%$ constant I is being correctly estimated, whereas at other times a combination of ZP is approximated for the constant I load. The dynamic simulation data seem to improve the estimation of the ZIP combinations during certain intervals compared to the estimation from steady-state measurements alone. By exploiting this periodicity of improved estimation results, the LSE was improved by using only those portions of lower voltage data that produce increased accuracy. Fig. 11 shows the updated estimation results for the $100 \%$ constant I scenario (corresponding to Fig. 10), where the estimation results are significantly improved by exploiting the periodicity (and letting the estimations be at the previous estimated values in between the periodic voltage fluctuations).
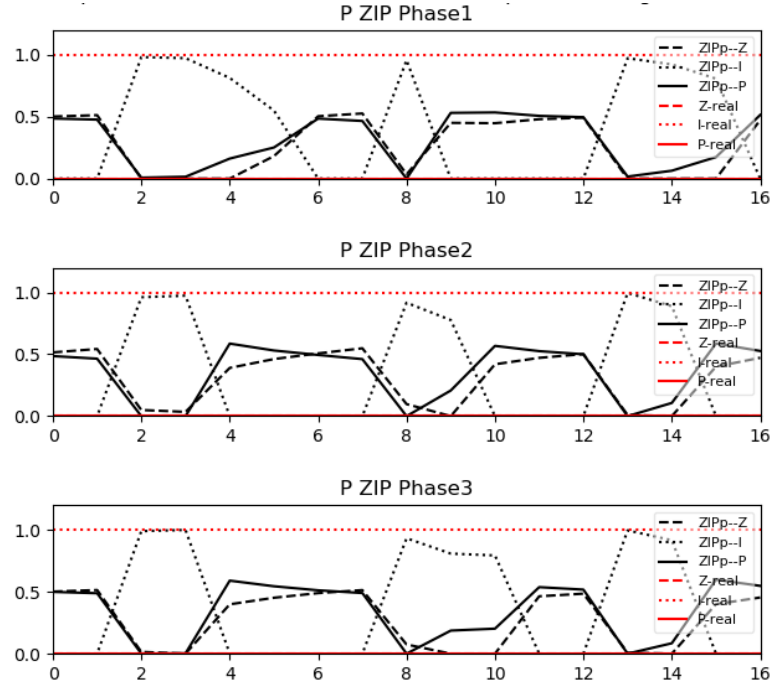

Fig. 10 100\% constant I real power: load 671 estimation results
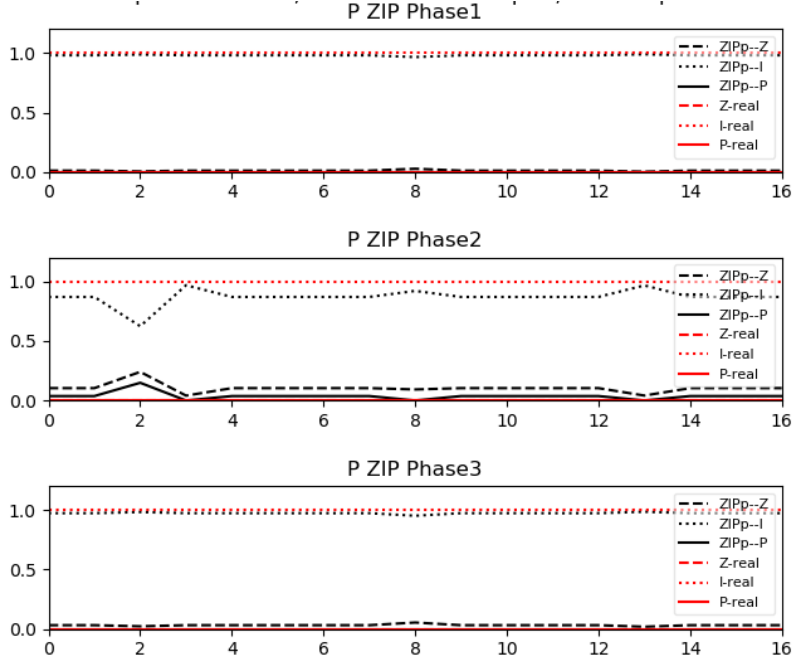

Fig. $11100 \%$ constant I real power: load 671 estimation with periodic sampling of dynamics (y-axis: ZIP proportion, $x$-axis: estimation windows) 
Similarly, Fig. 12 shows the estimation results for a ZIP combination scenario of $[0.3,0.1,0.6]$ ratio. Again, the estimation is better during the first few estimation windows because the lower voltage phenomena is captured (see Fig. 9) when the voltage is less than 0.9 p.u. By capturing lower voltage excursions (as one might see during fault conditions), the load estimations will improve. The exercises in this paper, however, indicate that even during normal load disturbance events (and possibly other switching and tap changing events that could produce lower voltage phenomena), the sliding window of power-voltage data can be used to estimate the load components using online algorithms.
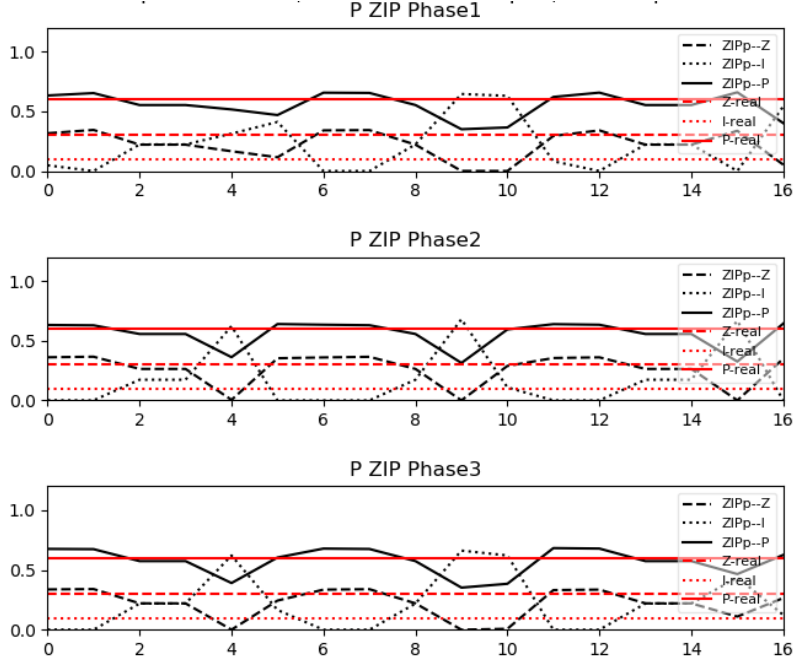

Fig. 12 ZIP combination $[0.3,0.1,0.6]$ : load 671 real power estimation (y-axis: ZIP proportion, $x$-axis: estimation windows)

\section{CONCLUSION}

This paper investigated the LSE method's performance for static ZIP load model estimation in distribution systems in an online manner using the sliding window technique of synthetic measurement data. The study was done using the IEEE 13node system. Synthetic measurement data at varying time intervals (namely, 15-min, $5 \mathrm{~s}$, and intra-seconds resolution) were generated to mimic different sensor characteristics (namely, AMI, SCADA, and microPMUs). The highest time resolution data were produced using the dynamic simulation of distribution networks (Gridlab-D delta mode), and QSTS simulation in OpenDSS was used to generate steady-state data (lower time resolution data).

The LSE method was able to differentiate $\mathrm{Z}$ and $\mathrm{P}$ load characteristics well; however, the performance for the I load was not good (in $100 \%$ of the I and combinations of ZI, IP, ZIP scenarios). The $100 \%$ I load estimated was improved with a revised version of the LSE method (called individual component LSE, $i L S E$ ). It was also found that a larger window size of data with a higher sampling rate (i.e., higher resolution sensor data) generally helped to improve the estimation results (because they can capture finer voltage variations); however, the LSE results using steady-state data indicated the need for lower voltage phenomena data to better differentiate between ZIP load characteristics. Therefore, dynamic simulation data subject to load disturbances were used, and the LSE estimation improved. Capturing such lower voltage phenomena (from fault events and other normal condition events, such as switching, tap changing, and abrupt load changes) in distribution systems offers a viable means of performing online static load modeling estimation. In future work, these methods will be validated by using real micro PMU data from power distribution networks and further extending the methods to composite load modeling.

\section{ACKNOWLEDGMENT}

This work was authored in part by the National Renewable Energy Laboratory (NREL), operated by Alliance for Sustainable Energy, LLC, for the U.S. Department of Energy (DOE) under Contract No. DE-AC36-08GO28308. Funding was provided by NREL's Laboratory Directed Research and Development (LDRD) program and the DOE Office of Electricity (OE) (FOA 0001616). The views expressed herein do not represent the views of the DOE or the Government.

\section{REFERENCES}

[1] Y. Ge, et. al., "An Event-Oriented Method for Online Load Modeling Based on Synchrophasor Data," IEEE Trans. Smart Grid, vol. 6, no. 4, pp. 2060-2068, 2015.

[2] A. Bokhari et al., "Experimental determination of the ZIP coefficients for modern residential, commercial, and industrial loads," IEEE Trans. Power Deliv., vol. 29, no. 3, pp. 1372-1381, 2014.

[3] North American Synchrophasor Initiative, Model Validation Using Phasor Measurement Unit Data, NASPI Technical Report, Mar 2015

[4] Krishnan, V. and J. McCalley. "Role of Induction Motors in Voltage Instability and Coordinated Reactive Power Planning." Chap. 6 in Induction Motors - Modelling and Control Edited by Prof. Rui Esteves Arajo, ISBN 9789535108436, InTech Open Access, November 2012.

[5] WECC, System Model Validation, DOE NASPI Workshop, Oct 2013

[6] D. Chassin, et. al., "FIDVR Load Modeling Tool," DOE/OE Transmission Reliability Program, June 2015

[7] Tushar, et. al., "Online Estimation of Steady-State Load Models Considering Data Anomalies," IEEE Trans. Ind. Appl., vol. 54, no. 1, pp. 712-721, 2018.

[8] H. Bai, P. Zhang, and V. Ajjarapu, "A Novel Parameter Identification Approach via Hybrid Learning for Aggregate Load Modeling," IEEE Trans. Power Syst., vol. 24, no. 3, pp. 1145-1154, 2009.

[9] P. Banerjee, S. M. H. Rizvi, and A. K. Srivastava, "Data-Driven Static Load Model Parameter Estimation with Confidence Factor," IEEE Int. Conf. Probabilistic Methods Appl. to Power Syst., pp. 1-6, 2018.

[10] F. Tuffner, "GridLAB-D - Dynamic Simulation Capabilities," PNNL, WECC Model Validation Working Group, Salt Lake City, Utah January $24,2018$.

[11] C. Wang, et. al., "Robust Time-Varying Parameter Identification for Composite Load Modeling," IEEE Trans. Smart Grid, vol. 3053, 2017.

[12] K. Wang, H. Huang, and C. Zang, "Research on time-sharing ZIP load modeling based on linear BP network," Proc. 2013 5th Int. Conf. Intell. Human-Machine Syst. Cybern. IHMSC, vol. 1, pp. 37-41, 2013.

[13] The Scipy community, "scipy.optimize.lsq linear - SciPy v0.18.1 Reference Guide." Available: https://docs.scipy.org/doc/scipy0.18.1/reference/generated/scipy.optimize.lsq_linear.html.

[14] Pacific Northwest National Laboratory, "GridLAB-D: A Unique Tool to Design the Smart Grid," 2012. [Online]. Available: http://www.gridlabd.org/brochures/20121130_gridlabd_brochure.pdf.

[15] Pacific Northwest National Laboratory, "Dev:Microgrids - GridLAB-D Wiki." [Online]. Available: http://gridlabd.sourceforge.net/wiki/index.php/Dev:Microgrids\#Interface_between machine_model_and_network_solution. [Accessed: 14-Nov-2018] 